\title{
Power Density Spectra of Gamma-Ray Bursts
}

\author{
Andrei M. Beloborodov ${ }^{1}$ \\ Stockholm Observatory, SE-133 36 Saltsjöbaden, Sweden
}

\begin{abstract}
Power density spectra (PDSs) of long gamma-ray bursts provide useful information on GRBs, indicating their self-similar temporal structure. The best power-law PDSs are displayed by the longest bursts $\left(T_{90}>100 \mathrm{~s}\right)$ in which the range of self-similar time scales covers more than 2 decades. Shorter bursts have apparent PDS slopes more strongly affected by statistical fluctuations. The underlying power law can then be reproduced with high accuracy by averaging the PDSs for a large sample of bursts. This power-law has a slope $\alpha \approx-5 / 3$ and a sharp break at $\sim 1 \mathrm{~Hz}$.

The power-law PDS provides a new sensitive tool for studies of gamma-ray bursts. In particular, we calculate the PDSs of bright bursts in separate energy channels. The PDS flattens in the hard channel $(h \nu>300 \mathrm{keV})$ and steepens in the soft channel $(h \nu<50 \mathrm{keV})$, while the PDS of bolometric light curves approximately follows the $-5 / 3$ law.

We then study dim bursts and compare them to the bright ones. We find a strong correlation between the burst brightness and the PDS slope. This correlation shows that the bursts are far from being standard candles and dim bursts should be intrinsically weak. The time dilation of dim bursts is probably related to physical processes occurring in the burst rather than to a cosmological redshift.

Finally, we test the internal shock model against the observed PDS. We demonstrate how the model can reproduce the $-5 / 3$ power law.
\end{abstract}

\section{Introduction}

The light curves of gamma-ray bursts (GRBs) typically have many random peaks and in spite of extensive statistical studies the temporal behavior of GRBs remains a puzzle. Contrary to the complicated diverse behavior in the time domain, long GRBs show a simple behavior in the Fourier domain (Beloborodov, Stern, \& Svensson 1998). Their PDS is a power-law of index $\alpha \approx-5 / 3$ (with a break at $\sim 1 \mathrm{~Hz}$ ) plus standard (exponentially distributed) statistical fluctuations superimposed onto the power law. The $-5 / 3$ slope and the $1 \mathrm{~Hz}$ break characterize the process randomly generating the diverse light curves of GRBs. Intriguingly, the PDS slope coincides with the Kolmogorov law.

\footnotetext{
${ }^{1}$ Also at the Astro-Space Center of Lebedev Physical Institute, Profsoyuznaya 84/32, 117810
} Moscow, Russia 
The power-law behavior is seen in individual bursts (we illustrate this in Section 3) and may provide a clue to the nature of GRBs. On the other hand, this behavior can be used as a tool in the studies of GRBs. In particular, one can study the PDS in separate energy channels (see Section 6). We quantify the difference of the temporal structure between the channels in terms of the PDS slope and compare the results with previous studies of the autocorrelation function $(\mathrm{ACF})$ in the separate channels. In Section 7, we address dim GRBs and compare them to the bright ones. Then, in Section 8, we simulate the observed PDS with the internal shock model. The results are discussed in Section 9.

\section{Data Analysis}

\subsection{The Sample}

In our analysis we use GRB light curves with $64 \mathrm{~ms}$ resolution obtained by BATSE in the four LAD energy channels, I-IV: (I) $20-50 \mathrm{keV}$, (II) $50-100$ $\mathrm{keV}$, (III) $100-300 \mathrm{keV}$, and (IV) $h \nu>300 \mathrm{keV}$. The background is subtracted in each channel using linear fits to the $1024 \mathrm{~ms}$ data.

Long bursts are of particular interest since their internal temporal structure can be studied by spectral analysis over a larger range of time scales. We choose bursts with durations $T_{90}>20 \mathrm{~s}$ where $T_{90}$ is the time it takes to accumulate from $5 \%$ to $95 \%$ of the total fluence of a burst summed over all the four channels, $\mathrm{I}+\mathrm{II}+\mathrm{III}+\mathrm{IV}$. Hereafter, we measure the brightness of a burst by its peak count rate, $C_{\text {peak }}$, in channels II+III. We analyze bursts with $C_{\text {peak }}>100$ counts per time-bin, $\Delta t=0.064 \mathrm{~s}$. This condition coupled with the duration condition, $T_{90}>20 \mathrm{~s}$, gives a sample of 559 GRBs. The sample still contains GRBs with low fluence, which are not good for Fourier analysis. We exclude bursts with fluences $\Phi<32 \Delta t C_{\text {peak }}$. The resulting sample contains 527 bursts.

\subsection{PDS Calculation}

We calculate the Fourier transform, $C_{f}$, of each light curve, $C(t)$, using the standard Fast Fourier Transform method. The power density spectrum, $P_{f}$, is given by $P_{f}=\left(C_{f} C_{f}^{*}+C_{-f} C_{-f}^{*}\right) / 2=C_{f} C_{f}^{*}$ since $C(t)$ is real. The Fourier transform is calculated on a standard grid with a time bin $\Delta t=64 \mathrm{~ms}$ and a total number of bins $N_{\text {time }}=2^{14}$, which corresponds to a total time $T \approx 1048$ s since the trigger time. The light curve of each burst is considered in its individual time window $\left(t_{1}, t_{2}\right)$. In the calculations of the PDS, we extend the time interval to $(0, T)$ by adding zeros at $\left(0, t_{1}\right)$ and $\left(t_{2}, T\right)$. See Beloborodov, Stern, \& Svensson (1999) for details of the data analysis.

Poisson noise in the measured count rate affects the light curve on short time scales, and, correspondingly, affects the PDS at high frequencies. The Poisson noise has a flat spectrum, $P_{f} \propto f^{0}$, introducing the "Poisson level", $P_{0}$, in a PDS. This level equals the burst total fluence including the background in the considered time window. The power spectrum above this level displays the intrinsic variability of the signal (but see Section 5 for the time-window effects). We calculate the individual Poisson level for each burst and subtract it from the burst PDS. 


\section{Individual Bright Bursts}

The brightest and longest bursts are the best ones for Fourier analysis. In this section we study the four brightest bursts with $T_{90}>100 \mathrm{~s}$. They have trigger numbers \# 2156 (GRB 930201), 2856 (GRB 940302), 3227 (GRB 941008), and 6472 (GRB 971110).

We take the light curves, $C(t)$, summed over channels II and III, in which the signal is strongest. To simplify the comparison of different bursts, we take peaknormalized light curves. Their Fourier transform, $C_{f}$, is therefore normalized by $C_{\text {peak }}$, and the PDS, $P_{f}=C_{f} C_{f}^{*}$, is normalized by $C_{\text {peak }}^{2}$.

The four peak-normalized light curves and their PDSs are shown in Figure 1 (we smooth the PDSs on the scale $\Delta \log f=0.04$ before plotting). The light curves are very different while the PDSs are similar. They can be described as a single power law, $\log P_{f}=A+\alpha \log f(A \approx 1$ and $\alpha \approx-1.5)$ with super-imposed fluctuations $\Delta P_{f} / P_{f} \sim 1$. In spite of the large $\Delta P_{f}$, the power-law behavior can be seen in each burst due to the power-law extending over more than two decades in frequency.

The presence of an underlying power law is an interesting feature of the GRB temporal behavior. A possible way to subtract it from the noisy individual PDSs is to take the average PDS over a sample of long GRBs. Then the fluctuations affecting each individual PDS tend to cancel each other and one can see the power law. The average of the four PDSs is plotted in Figure 2. One can see that the amplitude of irregularities is reduced after the averaging and one can measure the slope of the resulting PDS. The best power-law fit in the range $-2.0<\log f<0.2$ gives $\log P_{f}=1.25-1.45 \log f$.

The PDS analysis was previously performed for a number of individual bursts. Belli (1992), for example, analyzed GRBs detected by the Konus experiment onboard the Soviet probes Venera 11 and Venera 12, and Giblin et al. (1998) studied individual BATSE bursts. The power-law PDS can be observed in their longest complex bursts as well.

When turning to GRBs with relatively modest durations, $T_{90} \sim 20-100 \mathrm{~s}$, one finds it more difficult to see the power law in individual bursts. The statistical fluctuations, $\Delta P_{f} / P_{f} \sim 1$, significantly affect the apparent PDS slope derived from the available range of frequencies, which is limited by $\sim T_{90}^{-1}$. However, having a large number of bursts allows one to study the PDS by averaging over the sample.

\section{The Average PDS}

In most of the GRB models (e.g., in the internal shock model) different bursts are produced by one physical mechanism of stochastic nature, i.e., an individual burst is a random realization of the same standard process. Stern \& Svensson (1996) suggested an empirical pulse-avalanche model of this type. They showed that near-critical avalanches reproduce the statistical characteristics of GRBs as well as their extreme diversity. With such an approach, individual GRBs are like pieces of a "puzzle", and the features of the standard engine can be probed with statistical methods applied to a sufficiently large ensemble of GRBs. 

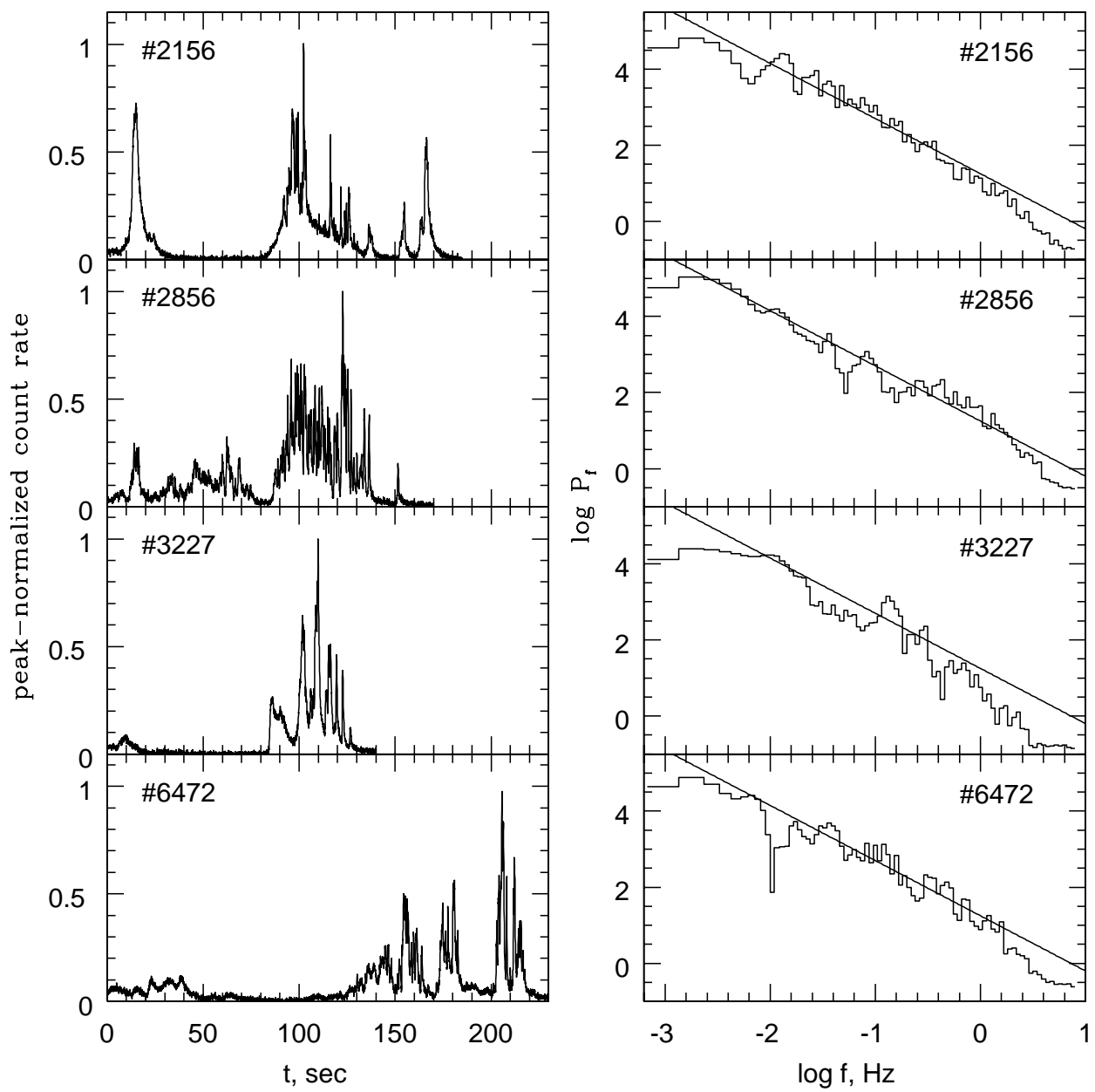

Figure 1. The light curves (in channels II+III) and their PDSs for the four brightest bursts in the sample with $T_{90}>100 \mathrm{~s}$. The light curves are taken peak-normalized and, correspondingly, the PDSs are normalized by $C_{\text {peak }}^{2}$. Since the bursts are very bright, their Poisson levels (normalized by $C_{\text {peak }}^{2}$ ) are very low, $P_{0} \approx 10^{-4}$. The straight lines show the fit to the average of the 4 PDSs, $\log P_{f}=1.25-1.45 \log f$ (see Fig. 2). 


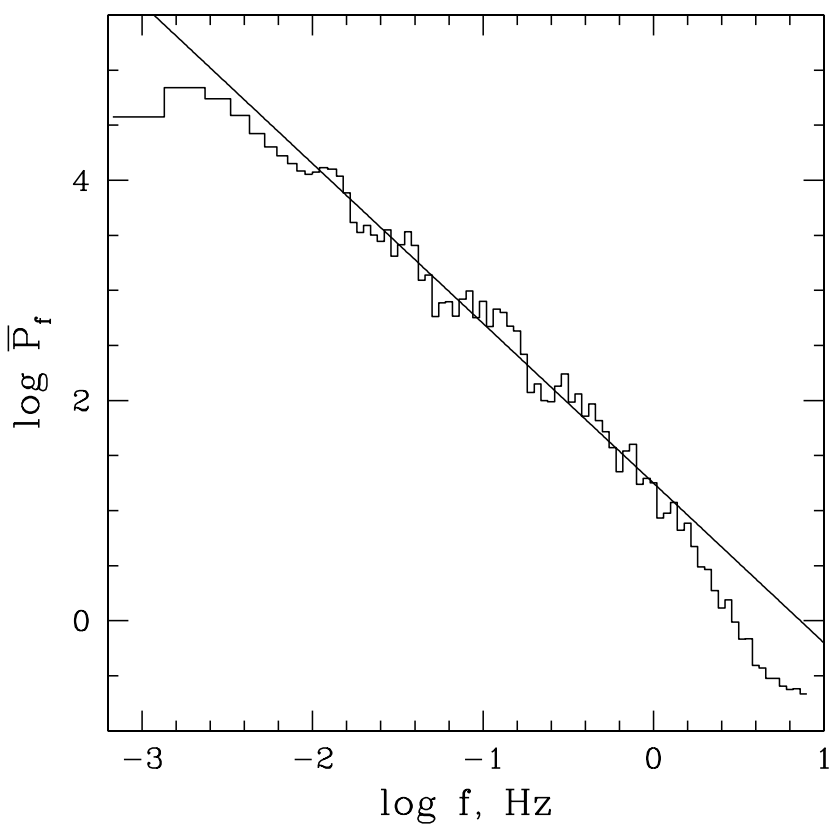

Figure 2. The average of the 4 PDSs shown in Figure 1. The line shows the best power-law fit, $\log P_{f}=1.25-1.45 \log f$, in the range $-2.0<\log f<0.2$.

The simplest statistical characteristic of the PDS is the average PDS, $\bar{P}_{f}$. The averaging means that we sum up the PDSs of individual bursts with some weights (normalization) and divide the result by the number of bursts in the sample. If no normalization is employed, then the brightest bursts strongly dominate $\bar{P}_{f}$, and their individual fluctuations lead to strong fluctuations in $\bar{P}_{f}$. The PDSs of relatively weak bursts are suppressed proportionally to $C_{\text {peak }}^{-2}$ and they are lost in the averaging. A normalization procedure is thus needed to increase the weight of relatively weak bursts in the sample. We prefer the peak-normalization because:

(i) The amplitude of the resulting PDS is practically independent of the burst brightness (see, e.g., Fig. 1).

(ii) The fluctuations in $\bar{P}_{f}$ turn out to be minimal and the best accuracy of the slope is achieved. The distribution of individual $P_{f}$ around $\bar{P}_{f}$ follows a standard exponential law (see Beloborodov et al. 1998), and the amplitude of fluctuations in $\bar{P}_{f}$ is given by a simple formula, $\Delta \bar{P}_{f} / \bar{P}_{f} \sim N^{-1 / 2}$, where $N$ is the number of bursts in the sample.

The averaging procedure is the following. (1) For each burst we first determine its individual Poisson level. (2) We determine the peak, $C_{\text {peak }}$, of the light curve, $C(t)$, with the background subtracted. (3) We normalize the light curve to its peak. (4) We calculate the PDS of the normalized light curve. (5) We normalize the Poisson level by $C_{\text {peak }}^{2}$ and subtract it from the PDS. (6) We sum 

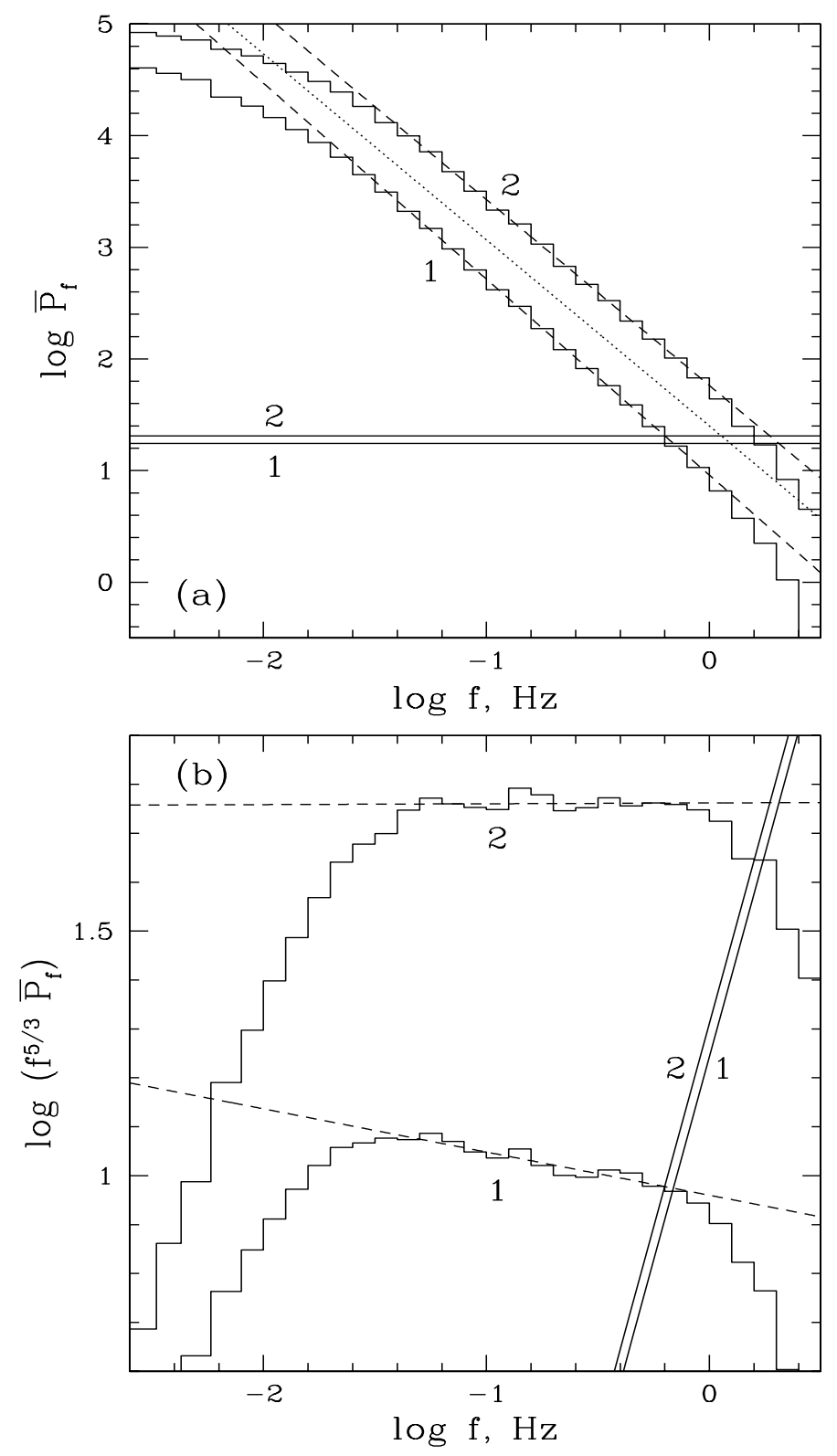

Figure 3. The average PDS (in channels II+III) for the full sample of 527 GRBs. (a) - The histograms marked as 1 and 2 correspond to the peak-normalization and $\sqrt{\Phi}$-normalization, respectively. The horizontal lines show the corresponding normalized Poisson levels averaged over the sample. Dashed lines are the best power-law fits in the range $-1.4<\log f<-0.1: \log \bar{P}_{f}=0.96-1.75 \log f$ in the peak-normalized case and $\log \bar{P}_{f}=1.76-1.67 \log f$ in the $\sqrt{\Phi}$-normalized case. Dotted line shows the $-5 / 3$ slope. (b) - Same as (a) except that now we plot $\bar{P}_{f}$ multiplied by $f^{5 / 3}$. 
up the resulting PDSs over the sample and divide by the number of bursts. The resulting average PDS, $\bar{P}_{f}$, for the 527 peak-normalized light curves in channels (II+III) is shown in Figure 3.

For comparison, we also plot the average PDS with the $\sqrt{\Phi}$-normalization, where $\Phi$ is the burst fluence excluding the background. Each light curve is then normalized by $\sqrt{\Phi}$, and its PDS (and its Poisson level) is normalized by $\Phi$. This normalization gives different weights (the weights of dim bursts are reduced as compared to the peak-normalization). The resulting average PDS has a different amplitude. Nevertheless, $\bar{P}_{f}$ again follows a power-law with approximately the same slope. This provides evidence that the self-similar behavior with $\alpha \approx$ $-5 / 3$ is an intrinsic property of GRBs, rather than an artefact of the averaging procedure. This interpretation is also supported by the fact that we observe the power-law in the longest individual bursts. When comparing individual PDSs, $P_{f}$, with the peak-normalized $\bar{P}_{f}$, one sees that $P_{f}$ is exponentially distributed around $\bar{P}_{f}$, and the distribution is self-similar with respect to shifts in $f$.

The power-law fit to $\bar{P}_{f}$ in the range $-1.4<\log f<-0.1$ gives $\alpha=-1.75$ for the peak-normalized bursts, and $\alpha=-1.67$ for $\sqrt{\Phi}$-normalized bursts. Note that the slope in the peak-normalized case is different from -1.67 reported in Beloborodov et al. (1998). This change is caused by that we have extended the sample to smaller brightnesses (see Section 7).

The deviation from the power-law at the low-frequency end is due to the finite duration of bursts. At the high frequency end, there is a break at $\sim$ $1 \mathrm{~Hz}$. The break is observed in the brightest GRBs even without subtracting the Poisson level (see Beloborodov et al. 1998 and the top panel in Fig. 7). Note that the break position is the same for the peak-normalized and $\sqrt{\Phi}$-normalized bursts. It stays the same in the separate energy channels (see Fig. 5) and does not depend on the Poisson level. One may also see the break in individual long bursts (Figs. 1 and 2). The break is far too sharp to be explained as an artefact of the $64 \mathrm{~ms}$ time binning, which suppresses the PDS by a factor of $[\sin (\pi f \Delta t) /(\pi f \Delta t)]^{2}$ where $\Delta t=64 \mathrm{~ms}$ is the time bin (cf. van der Klis 1989).

\section{The Effects of Finite Signal Duration}

Fourier analysis was designed for physical problems dealing with linear differential equations. For example, it is usually applied to small perturbations above a given background solution. The Fourier power spectrum is also commonly used in the temporal studies of long signals or noises, e.g., in persistent astrophysical sources. By contrast, GRBs are strongly non-linear and short signals. The typical number of BATSE time-bins $(\Delta t=64 \mathrm{~ms})$ in a long GRB is a few $\times 10^{3}$. Do the effects of finite duration (i.e., time-window effects) strongly affect the measured PDS?

The issue is illustrated in Figure 4. We prepared an artificial long signal with $\bar{P}_{f} \propto f^{\alpha}$ and exponentially distributed $P_{f}$, i.e., the probability to detect $P_{f}$ at a given $f$ is proportional to $\exp \left(-P_{f} / \bar{P}_{f}\right)$. The phase structure was taken to be Gaussian (random). The signal duration is $T_{0}=2^{17} \Delta t \approx 8400 \mathrm{~s}$. Then, we cut the long signal into 64 pieces of equal length $t_{0}=2^{11} \Delta t \approx 130 \mathrm{~s}$. We thus get 64 random short signals, each is a random realization/fragment of the same 


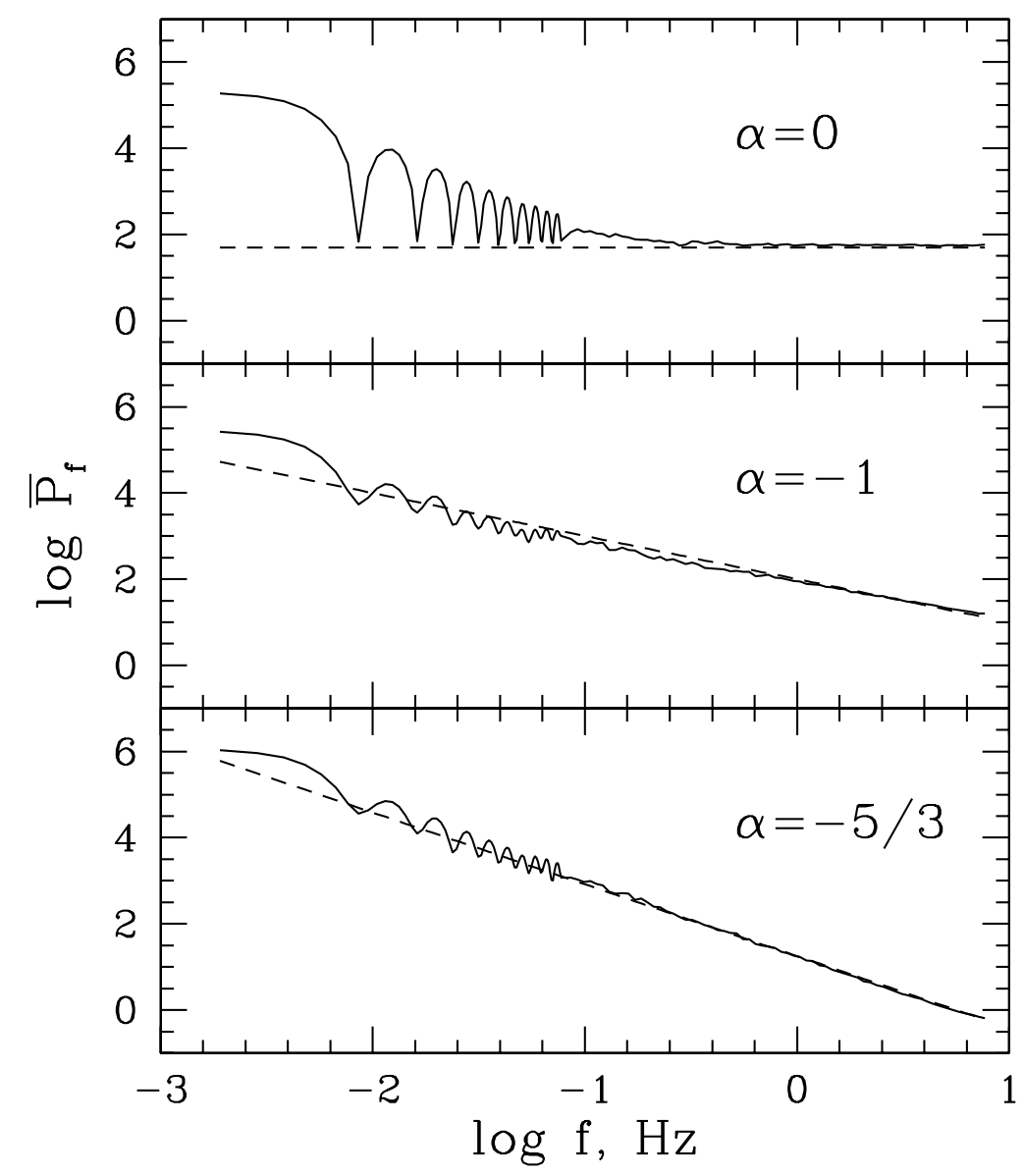

Figure 4. The average PDS, $\bar{P}_{f}$, for the 64 fragments of noise with a power-law PDS of index $\alpha$ (see text for details). Dotted lines show the original PDS slope. The deviation of $\bar{P}_{f}$ from this slope is due to the time-window effects.

stationary process characterized by the index $\alpha$. Analogously to our analysis of real GRBs, we normalize each signal to its peak and add zeros up to $T=2^{14} \Delta t$ (our standard grid). Then we calculate the average PDS, $\bar{P}_{f}$, for the 64 artificial signals. The result is shown in Figure 4 for the three cases: $\alpha=0$ (Poisson), $\alpha=-1$ (flicker), and $\alpha=-5 / 3$ (Kolmogorov).

One can see that the time-window effects are strong in the case $\alpha=0$. The Poisson signal is roughly constant on large time scales. As a result, at modest $f, \bar{P}_{f}$ is just the power spectrum of the $t_{0}$-window. The PDS shape in the case $\alpha=0$ can easily be calculated analytically. The Poisson signal can be modelled as a sum of $N$ elementary narrow pulses ( $\delta$-functions), with each pulse having equal probability to appear at any moment $t$ within the interval $\left(0, t_{0}\right)$. The Fourier transform of one random realization $\left(t_{1}, \ldots, t_{N}\right)$ of the signal is $C_{f}=$ $\Sigma \exp \left(-i 2 \pi f t_{k}\right)$, and $P_{f}=C_{f} C_{f}^{*}$. After averaging over many realizations, one 
gets

$$
\bar{P}_{f}=N+\frac{N(N-1) \sin ^{2}\left(\pi f t_{0}\right)}{\left(\pi f t_{0}\right)^{2}} .
$$

The time-window effects are dominant at $f<\sqrt{N} /\left(\pi t_{0}\right)$. At $f>\sqrt{N} /\left(\pi t_{0}\right), \bar{P}_{f}$ approaches the Poisson level, $N$.

By contrast, in the case $\alpha=-5 / 3$, we have large-amplitude variations in the signal on all time scales. The average PDS of the 64 short signals then reproduces well the intrinsic PDS slope throughout the whole range of frequencies, down to $f \sim t_{0}^{-1}$ (see bottom panel in Fig. 4). Hence, the power law we observe in the average PDS of GRBs can be interpreted as that GRBs are random short realizations of a standard process which is characterized by the PDS slope $\alpha \approx-5 / 3$. Note, however, that the power spectrum does not provide a complete description of the signal since the phase structure is not considered.

\section{The PDSs and the ACFs in Channels I, II, III, IV}

What does the average PDS look like in separate energy channels I, II, III, and IV? The signal to noise ratio is low in channel I and especially in channel IV. The number of bursts for which good PDSs can be obtained in channels I and IV is therefore limited to the brightest bursts. In order to compare the PDSs in different channels, we choose a sample of bursts with $C_{\text {peak }}>500$ counts/bin (in channels II+III), which contains 152 bursts.

The burst analysis is now performed in each channel separately. We determine the Poisson level of a burst in each channel, $P_{0}^{i}$, and find the peak of the light curve, $C_{\text {peak }}^{i}$, where $i=\mathrm{I}$, II, III, IV. Then we perform the peaknormalization: $C^{i}(t) \rightarrow C^{i}(t) / C_{\text {peak }}^{i}$ and $P_{0}^{i} \rightarrow P_{0}^{i} /\left(C_{\text {peak }}^{i}\right)^{2}$.

The resulting average PDSs in channels I-IV are shown in Figure 5. The differences in the slopes are clearly seen. We fitted the PDSs by power laws, $\log \bar{P}_{f}=A+\alpha \log f$, in the range $-1.6<\log f<0$. Channel IV is fitted in the range $-1.3<\log f<-0.1$. $A$ and $\alpha$ of the best fits are listed in Table 1 .

Table 1.

\begin{tabular}{ccccc}
\hline \hline & & & & \\
Channel & I & II & III & IV \\
$\alpha$ & -1.72 & -1.67 & -1.60 & -1.50 \\
$A$ & 1.03 & 1.05 & 1.06 & 1.07 \\
\hline
\end{tabular}

The observed behavior can be briefly described as follows: The "red" power $\left(\bar{P}_{f}\right.$ at low $\left.f\right)$ decreases at high photon energies and increases at low photon energies. It should be compared with the well-known fact that the pulses in a GRB are more narrow in the hard channels (e.g., Norris et al. 1996). The hardness of emission varies dramatically during a burst and this leads to different temporal structure in different channels. E.g., pulses observed in the soft channels may be suppressed in the hard channels. One therefore could expect that the PDS has different slopes in different channels. Note that, typically, 


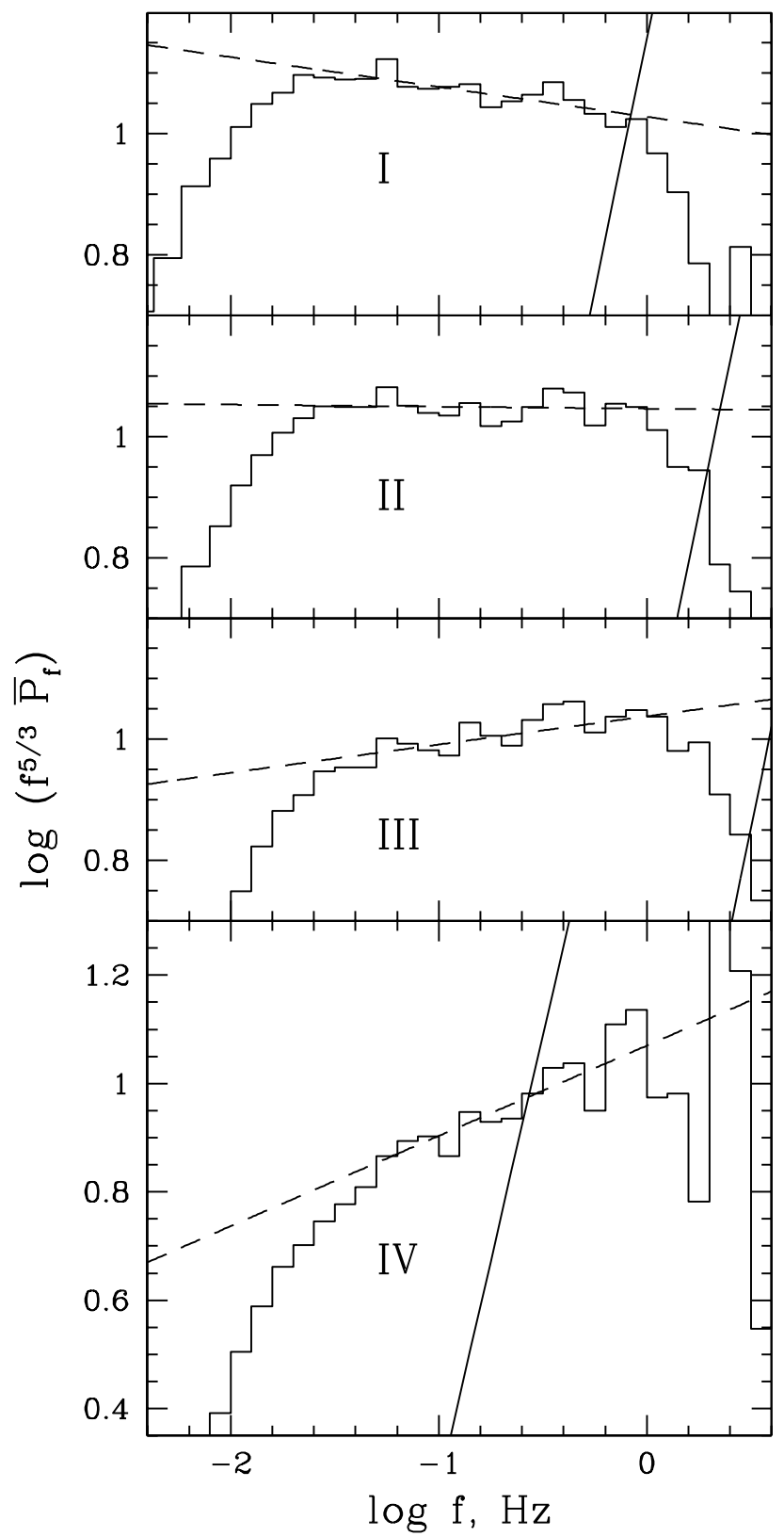

Figure 5. The average PDS for the sample of 152 GRBs in separate energy channels, I, II, III, and IV. Dashed lines show the best power-law fits (see Table 1). Solid lines show the Poisson level. 
most of the GRB energy is released in channels II+III, and the average PDS of bolometric light-curves approximately follows the $-5 / 3$ law.

In principle, the autocorrelation function $(\mathrm{ACF})$ contains the same information as the PDS, since one is the Fourier transform of the other (the WienerKhinchin theorem). In practice, the two are not completely equivalent because of the time-window effects and the presence of a noisy background. On modest time scales, $\lesssim 30 \mathrm{~s}$, the direct ACF calculation and the calculation via the Fourier transform of the PDS give the same result to within a few percent (we have calculated the ACF by both methods). The average ACF, $\bar{A}(\tau)$, for our sample of 152 bright GRBs is shown in Figure 6 in each of the four channels. The ACF gets narrow at high energies, in agreement with previous studies (Fenimore et al. 1995), except that our ACF is $\sim 2$ times wider as compared to that obtained by Fenimore et al. (1995). The ACF width averaged over the channels is in approximate agreement with that calculated for the bolometric light curves by Stern \& Svensson (1996).

Note that the average ACF is obtained by summing up the individual ACFs normalized to unity, i.e., $A(0)=1$. Recalling that the $\mathrm{ACF}$ is the Fourier transform of the PDS, one can see that this normalization is equivalent to the normalization of the light curve by $\sqrt{P_{\text {tot }}}$ where $P_{\text {tot }}=\int[C(t)]^{2} d t=\int P_{f} d f$ is the total power. This normalization is different from the peak-normalization we use in the calculations of the average PDS, i.e., we prescribe different weights to individual bursts when averaging the PDS and the ACF, respectively. Therefore, the average ACF is not the Fourier transform of the average PDS shown in Figure 5. This relation holds when the average PDS is calculated with the $\sqrt{P_{\text {tot }}}$-normalization.

The ACF in each channel is perfectly fitted by the stretched exponential: $\bar{A}(\tau)=\exp \left(-\left[\tau / \tau_{0}\right]^{\beta}\right)$ (see Figure 6 ). The parameters $\tau_{0}$ and $\beta$ are listed in Table 2. The index $\beta$ is related to the PDS slope $\alpha$ by the simple relation $\beta \approx-(1+\alpha)$.

Table 2.

\begin{tabular}{ccccc}
\hline \hline Channel & I & II & III & IV \\
$\beta$ & 0.73 & 0.67 & 0.62 & 0.6 \\
$\tau_{0}$ & 14.0 & 10.7 & 7.3 & 5.1 \\
\hline
\end{tabular}

The changing $\beta$ demonstrates that the ACF shape changes from channel to channel, as it should do since the PDS changes. As a first approximation, the $\mathrm{PDS}$ slope is equal to $-5 / 3$, and the ACF index $\beta \approx 2 / 3=5 / 3-1$.

The values of $\tau_{0}$ quantify the ACF width in different channels. The parameter $\tau_{0}$ is better defined as compared to measuring the ACF width at a certain level, e.g., at $e^{-0.5}$ of the maximum level, as was done in Fenimore et al. (1995). Nevertheless, the scaling of $\tau_{0}$ with energy is approximately the same, $\tau_{0} \propto E^{-0.4}$ where $E$ is the photon energy of the low energy boundary of the channel, see Fenimore et al. (1995). It should be stressed, however, that the stretching of $\tau_{0}$ is not related to the stretching of any physical time scale of intrinsic correlations during the burst. The ACF width, $\tau_{0}$, is rather related to the position of the 

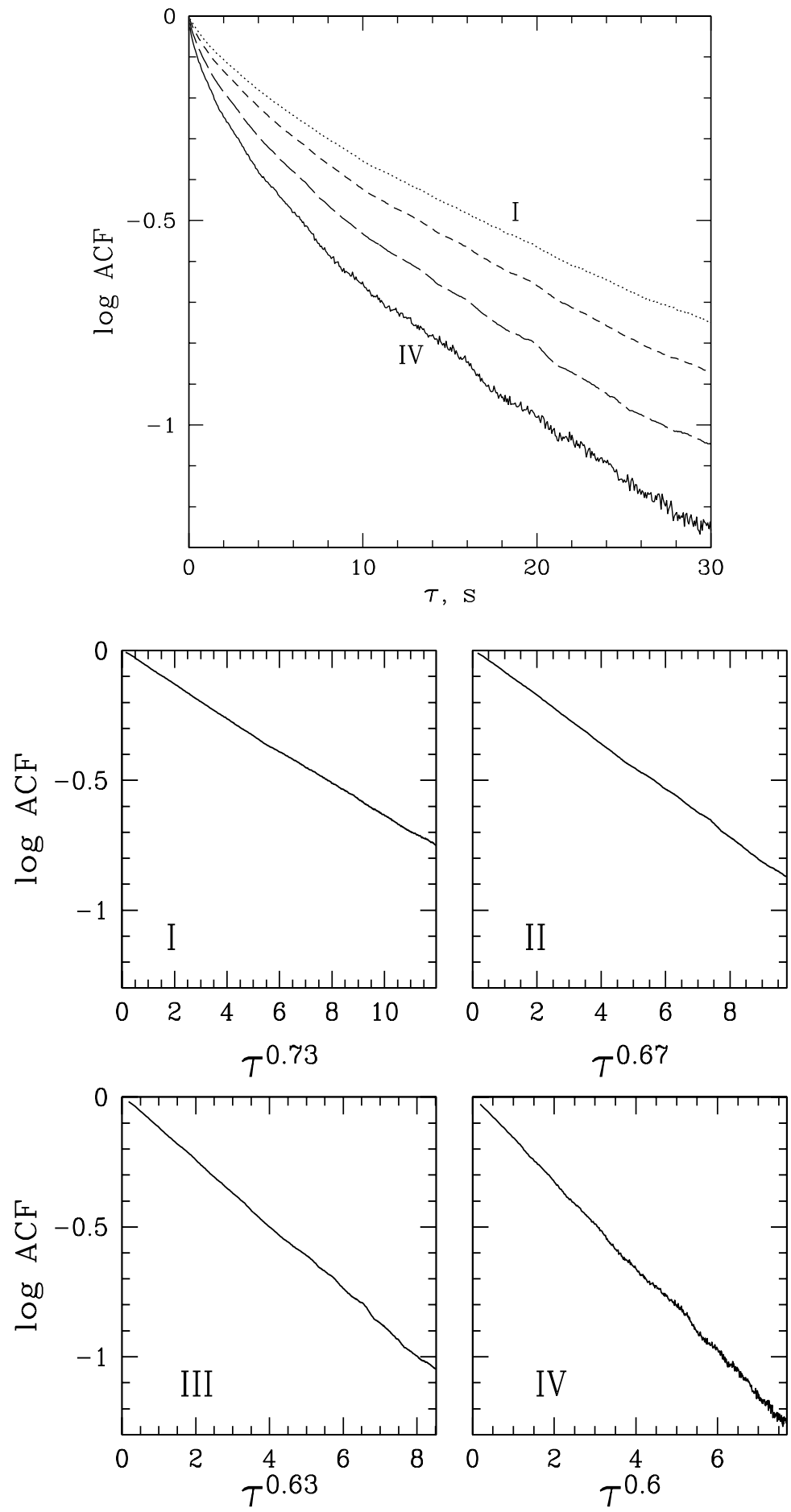

Figure 6. The average autocorrelation function for the sample of 152 bright bursts, in channels I-IV. 
breaks in the PDS, especially with the low frequency break, which in turn is determined by the burst duration. For an infinitely long signal with a power law PDS, $t_{0}$ would tend to infinity. This is natural since the power law behaviour implies self-similarity, i.e., the absence of any preferred time scale. Specific time scales are introduced only by the breaks in the PDS. We therefore have only two physical time scales in long GRBs: The first one is associated with the $1 \mathrm{~Hz}$ break and the second one is associated with the low frequency break due to the finite burst duration.

\section{Dim versus Bright Bursts}

Now we address the following question: Is there any correlation between the PDS slope and the burst brightness?

\subsection{PDS Slope Correlates with the Burst Brightness}

We take the full sample of 527 light curves in channels II+III and divide it into 3 groups of different brightnesses: (A) $C_{\text {peak }}>800$, (B) $300<C_{\text {peak }}<$ 800, and (C) $100<C_{\text {peak }}<300$. Group A contains 91 bursts, group B 222 bursts, and group C 214 bursts. We have calculated the average PDS for each group separately employing the peak-normalization. The results are presented in Figure 7. We fitted the average PDSs by power laws, $\log P_{f}=A+\alpha \log f$. The parameters $A$ and $\alpha$ of the best fits in the range $-1.5<\log f<-0.1$ are listed in Table 3 . We conclude that the average PDS of dim bursts gets markedly steeper.

Table 3.

\begin{tabular}{cccc}
\hline \hline & & & \\
group & $\mathrm{A}$ & $\mathrm{B}$ & $\mathrm{C}$ \\
$\alpha$ & -1.63 & -1.74 & -1.82 \\
$A$ & 1.04 & 0.98 & 0.91 \\
\hline
\end{tabular}

\subsection{Subtraction of the Poisson Level}

In the PDS calculations we subtracted the Poisson level, which is quite high for dim bursts (see Fig. 7). One therefore should address a technical question: How well is the intrinsic PDS restored after subtracting the Poisson level?

To investigate this issue we have performed the following test. We take the sample of 91 bright bursts (group A) and add a strong Poisson noise (with an average level of 25000 counts/bin) to each burst in the group. We thus increase artificially the Poisson level by two orders of magnitude. Alternatively, one may consider this procedure as a rescaling of bright bursts to a smaller brightness while keeping the Poisson background at the same level. Then, we analyze the artificial bursts in the same way as we did with the real dim GRBs. Note that even after subtraction of the time-averaged background, its Poisson noise strongly affects the signal and may create artificial peaks dominating the true 


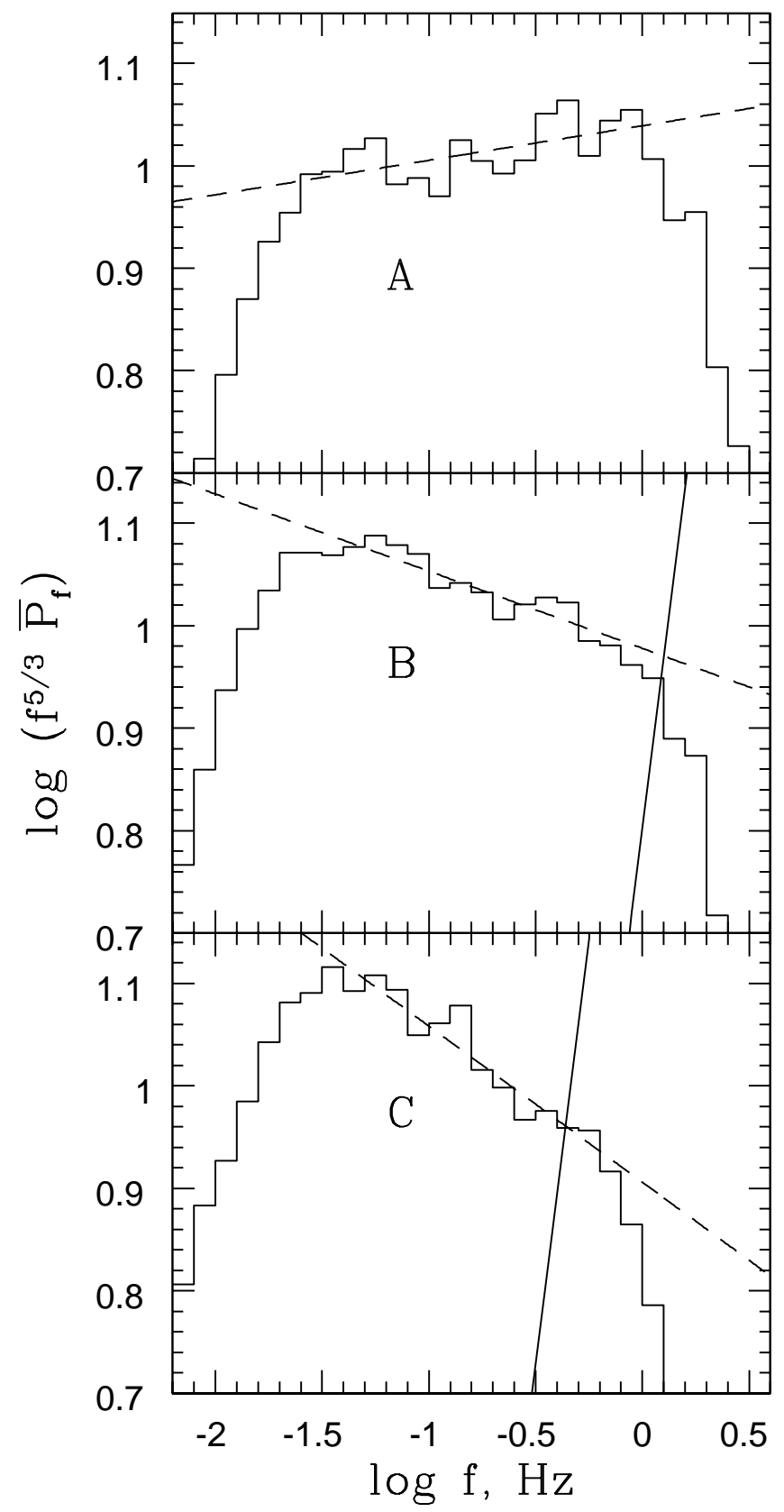

Figure 7. The average PDSs for the three brightness groups A, B, and C. Dashed lines show the power-law fits (see Table 3). Solid lines show the Poisson level. 


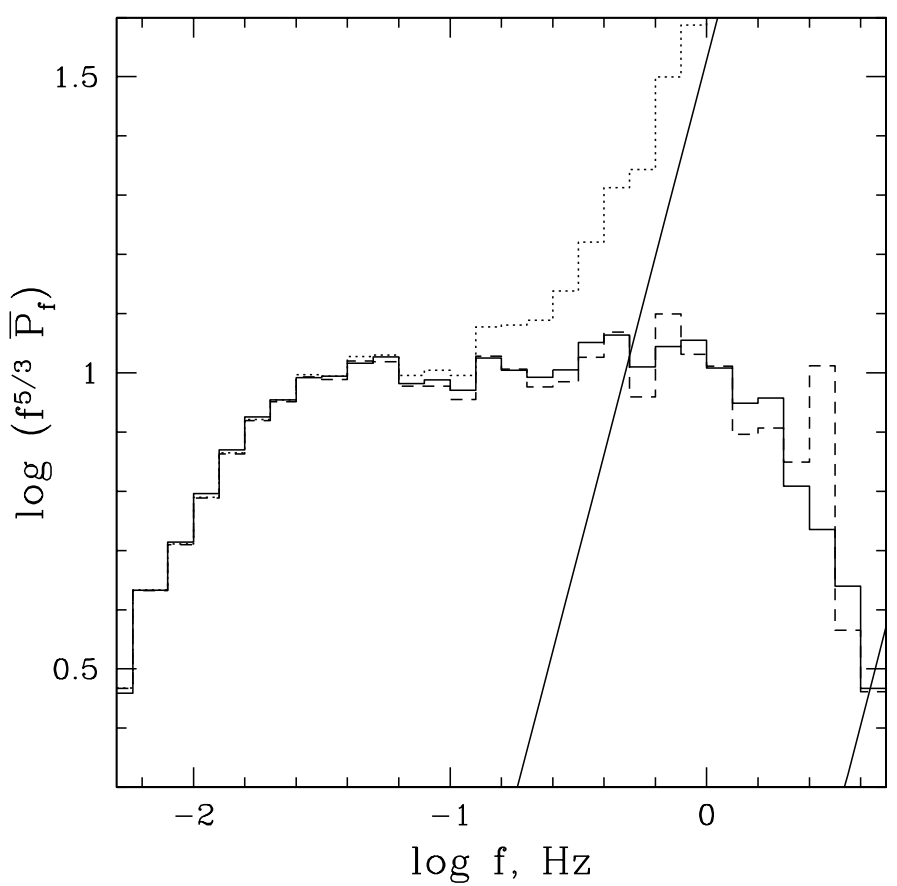

Figure 8. The average PDS of 91 artificial dim bursts created from group A of the brightest bursts (see Fig. 7). Solid histogram - the original PDS for group A. Dashed and dotted histograms - the average PDS of the artificial bursts with and without subtraction of the Poisson level, respectively. The two solid lines show the average Poisson levels for the original and the artificial bursts, respectively.

peak of the signal. For the artificial bursts, we know the true peak (which is the peak of the original bright burst). In real dim bursts we do not know the position of the true peak and employ the peak search scheme described in Stern, Poutanen, \& Svensson (1999).

The result is compared with the average PDS of the original sample in Figure 8. We find that the subtraction of the Poisson level allows one to restore the original PDS, $P_{f}$, at frequencies where $P_{f}$ is well below the Poisson level. Even the $1 \mathrm{~Hz}$ break remains present. We conclude that the high Poisson level is unlikely to affect significantly the measured PDS slope.

\section{The Internal Shocks Model}

A likely scenario of GRBs involves internal shocks in a relativistic outflow with a Lorentz factor $\Gamma \sim 10^{2}$ (see, e.g., Piran 1999 for a review). The shock develops when an inner faster shell of the outflow tries to overtake the previous slower shell. The pulses in a burst are then associated with collisions between the shells. The process was simulated numerically by Kobayashi, Piran, \& Sari (1997) and Daigne \& Mochkovitch (1998). 
We have performed analogous simulations to see what kind of PDS the model can produce. The details of the emission mechanism are still disputed (see, e.g., Mészáros \& Rees 1999). For simplicity, we assume that the energy of shocked plasma is instantaneously radiated. Then the pressure stays low and its dynamical effect is negligible, i.e., the matter is in free motion (coasting) everywhere in the outflow. As time goes on, the outflow mass gets concentrated in caustics that are thin spherically symmetric shells, and the density is reduced between the caustics. The caustics have different velocities; they can catch up with each other and merge with energy release.

We take the duration of the outflow, $T_{0}=\Delta / c=100 \mathrm{~s}$, where $\Delta$ is the distance between the leading and the back fronts of the outflow. The outflow becomes optically thin when it reaches a radius $R_{*} \sim 10^{13}-10^{14} \mathrm{~cm} \gg \Delta . R_{*}$ depends on the average density and Lorentz factor of the outflow; it is taken as a parameter in our simulations. We assume that the energy released at the optically thick stage is lost as a result of adiabatic expansion, and only the radiation produced at $R>R_{*}$ is observed. We also neglect the dynamical pressure effect of radiation trapped during the optically thick stage. This may be a reasonable approximation taking into account the modest radiative efficiency of internal shocks, which implies that the energy density of radiation is small compared to the matter kinetic energy.

The process of energy release is governed by the velocity and the density profiles of the outflow, which should be specified as initial conditions at some moment before the outflow reaches $R_{*}$. We prepare the initial conditions in the following way. At a given radius, $R_{0} \ll R_{*}$, we "inject" the first shell, then (after a delay $\delta t$ ) the second shell and so on, until the last shell. It corresponds to "sampling" the velocity and density through the outflow when it passes radius $R_{0}$. The initial state is thus represented by the Lorentz factors and masses of the discrete shells, $\gamma\left(t_{i}\right)$ and $m\left(t_{i}\right), i=1, \ldots, N$. Here $N$ is the total number of injected shells, $\delta t=t_{i+1}-t_{i}=T_{0} / N$ is the time scale initially separating the shells. In the simulations, we take $N=2 \times 10^{4}$. Details of the simulations are described in Beloborodov (1999).

The temporal structure of the injection, $\gamma\left(t_{i}\right)$ and $m\left(t_{i}\right)$, is the main issue of the problem. Usually, it was assumed to be a white noise, which leads to a wrong PDS of the simulated bursts. Trying to reconcile the model with the observed -5/3 PDS, Panaitescu, Spada, \& Mészáros (1999) took a correlated injection with a sinusoidal profile. It leads to extra "red" power. The PDS, however, still does not possess the self-similar structure that we observe in real GRBs. It appears to be important to have such a structure in the outflow before it becomes optically thin. We have studied three different regimes of $\gamma\left(t_{i}\right)$ and $m\left(t_{i}\right)$ : (1) white noise, (2) flicker noise, and (3) Kolmogorov noise, and calculated the resulting light curves and their PDSs.

The best agreement with the observed PDS is achieved if the initial density and/or the Lorentz factor have a Kolmogorov-like structure. In Figure 9, we show the result for the case where: (i) the injection Lorentz factor is randomly chosen between 50 and 150 (white noise), (ii) the injection density, $m\left(t_{i}\right)$, is a signal with the $-5 / 3$ PDS and random phase structure (Kolmogorov noise), and (iii) $R_{*}=10^{14} \mathrm{~cm}$. 

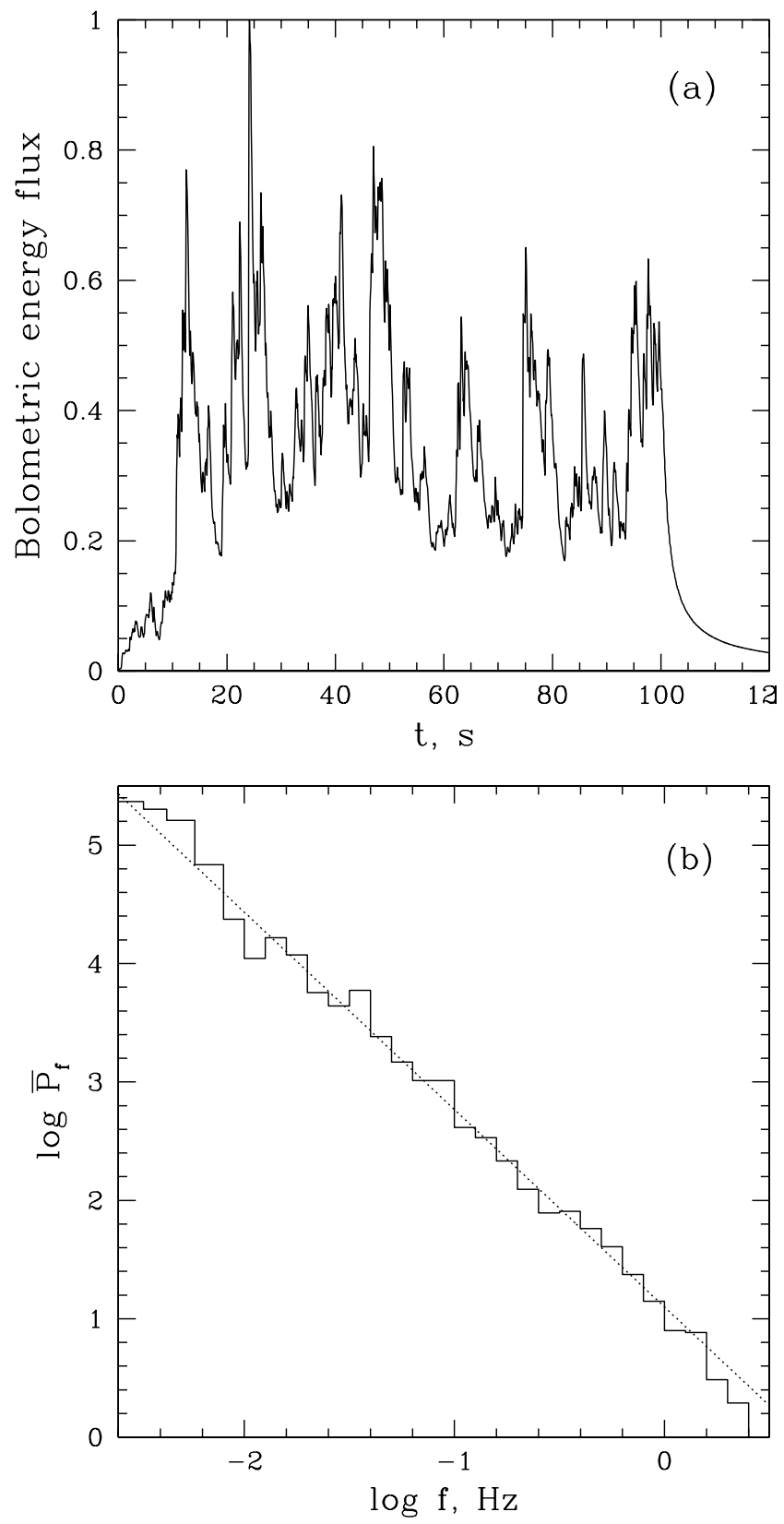

Figure 9. (a) - One example of the simulated light curves (bolometric energy flux as a function of time). (b) - The average PDS of 26 simulated bursts, each is produced by one realization of the random injection process described in the text. Dotted line shows the $-5 / 3$ slope. 
To the first approximation, the light curve seen by a distant observer goes in the same order as the injection, i.e., first the observer sees the emission from internal shocks in the leading part of the outflow and then receives radiation from deeper and deeper layers. Different parts of the outflow are causally disconnected during the main part of the emission time. It implies that the light from the back parts cannot overtake the leading front of the outflow during the formation of the signal, and radiation from the leading and back parts cannot come to the observer in the reverse order. The observed temporal structure thus conforms to the temporal structure of injection, and the resulting slope of the PDS is basically the same as prescribed to the flow at the inner boundary. For a more detail discussion see Beloborodov (1999).

\section{Discussion}

\subsection{Relation to the Average Time Profile}

The average time profile (ATP) of GRBs was found to follow the stretched exponential of index $1 / 3$ (Stern 1996). Is there any relation between the ATP and the average PDS? One should note two important differences between the ATP and the PDS studies: (i) the $-5 / 3$ PDS, though affected by the statistical fluctuations, is observed in individual bursts, while the ATP is a purely statistical property of a large sample of bursts; (ii) the ATP is constructed for GRBs of all durations (and only in this case it displays the perfect stretched exponential) while the PDS is studied for long bursts only. Nevertheless, both the ATP and the PDS characterize the stochastic process generating GRBs.

\subsection{The $1 \mathrm{~Hz}$ Break}

The sharpness of the break in the average PDS appears to be an important feature that constrains models of GRBs. If the signal is produced in the rest frame of a relativistic outflow then variations of the outflow Lorentz factor, $\Gamma$, from burst to burst would smear out the break. The sharpness of the break then implies a narrow dispersion of $\Gamma$, which appears to be unlikely.

Alternatively, the GRB variability may come from the central engine. The break in the PDS might then be related to a typical time scale, $\sim 1 \mathrm{~s}$, in the central engine.

Finally, one may associate the break with the dynamical time scale corresponding to the inner radius of the optically thin zone of the outflow, $R_{*}$. Then the variability on time scales shorter than $t_{*}=R_{*} / c \Gamma^{2}$ can be suppressed. In this case, however, one should explain why $t_{*}$ stays the same in different bursts.

\subsection{Dim GRBs}

It has often been hypothesized that dim bursts are at high cosmological redshifts. For instance, it is necessarily the case if GRBs have approximately the same intrinsic luminosity, the so-called "standard candle" hypothesis. We can test this hypothesis using the power spectrum analysis.

Suppose that the dim bursts are intrinsically the same as the bright ones. Then any difference in their average PDS should be due to a cosmological redshift. First consider the bolometric light curves assuming that their average PDS 
follows the $-5 / 3$ power law. It is easy to see that a redshift, $z$, will not change the PDS slope. As we normalize each bursts to its peak before the averaging, the effect of a redshift is just stretching the light curve in time, i.e., precisely the time dilation effect. This will lead to an increase in the net normalization of the PDS by a factor of $(1+z)^{1 / 3}$. The slope does not change since the dilation factor $(1+z)$ is the same for each time scale.

One should, however, recall that we observe bursts in a limited spectral interval. A redshift then implies a shift of the signal with respect to our spectral window. E.g., photons detected in channel III would originally have been emitted in channel IV. As we know from Section 6, the PDSs are different in different energy channels. Therefore, one expects that the PDS slope will change for redshifted GRBs.

We have seen in Section 6 that the PDS of bright bursts flattens in the hard channels. Hence, a redshift of the bright bursts must be accompanied by a flattening of the PDS. Contrary to this behavior, we observe that the PDS of dim bursts steepens. Hence, the evolution of the PDS with brightness is inconsistent with the standard candle hypothesis. It implies that the burst luminosity function is broad and dim bursts are intrinsically weak.

Evidence for a broad luminosity function is also found when looking at the isotropic luminosities of the bursts with measured redshifts. Note, however, that the differences in the apparent luminosities could be caused by orientation effects if GRBs are beamed. One should not therefore exclude that the total intrinsic luminosities are approximately the same. The different temporal structure of dim bursts may be an important fact in this respect. In particular, it suggests that the observed time dilation of dim bursts (e.g., Norris et al. 1994) may be caused mainly by physical processes occurring in the bursts rather than by a cosmological redshift. Note that the intrinsic difference of the temporal structure of $\operatorname{dim}$ GRBs was also found when studying their average time profile (see Stern et at. 1997). The rising part of the ATP does not change with decreasing brightness while the decaying part suffers from time dilation. This behavior is inconsistent with a cosmological time dilation which should apply equally to both parts of the ATP.

\subsection{Internal Shocks}

Further constraints on the internal shock model should be imposed by the observed dependence of $\alpha$ on photon energy. In particular, the detailed emission model studied by Panaitescu, Spada, \& Mészáros (1999) and Spada, Panaitescu, \& Mészáros (1999) can be tested. Besides, the phase structure of the signal, neglected so far, should be taken into consideration. In particular, the efficiency of the energy release depends on the degree of coherence of the internal shocks. More detailed analysis in this direction will be given elsewhere (Beloborodov 1999).

Acknowledgments. I thank A. F. Illarionov, I. Panchenko, J. Poutanen and R. Svensson for discussions. This research was supported by the Swedish Natural Science Research Council and RFBR grant 97-02-16975. 97-02-16975. 


\section{References}

Belli, B. M. 1992, ApJ, 393, 266

Beloborodov, A. M. 1999, in preparation

Beloborodov, A. M., Stern, B.E., \& Svensson, R. 1998, ApJ, 508, L25

Beloborodov, A. M., Stern, B. E., \& Svensson, R. 1999, in preparation

Daigne, F., \& Mochkovitch, R. 1998, MNRAS, 296, 275

Fenimore, E.E., In’t Zand, J. J. M., Norris, J.P., Bonnell, J.T., \& Nemiroff, R. J. 1995, ApJ, 448, L101

Giblin, T. W., Kouveliotou, C., \& van Paradijs, J. 1998, in AIP Conf. Proc. 428, Gamma-Ray Bursts, ed. C.A. Meegan, R. D. Preece, \& T. M. Koshut (New York: AIP), 241

Kobayashi, S., Piran, T., \& Sari, R. 1997, ApJ, 490, 92

Mészáros, P., \& Rees, M. 1999, ApJ, submitted (astro-ph/9908126)

Norris, J. P., Nemiroff, R. J., Bonnell, J. T., Scargle, J. D., Kouveliotou, C., Paciesas, W.S., Meegan, C. A., \& Fishman, G. J. 1996, ApJ, 459, 393

Norris, J.P., Nemiroff, R. J., Scargle, J.D., Kouveliotou, C., Fishman, G. J., Meegan, C. A., Paciesas, W.S., \& Bonnell, J. T. 1994, ApJ, 424, 540

Panaitescu, A., Spada, M., \& Mészáros, P. 1999, ApJ, 522, in press

Piran, T. 1999, Phys. Rep., 314, 575

Spada, M., Panaitescu, A., \& Mészáros, P. 1999, ApJ, submitted (astro-ph/9908097)

Stern, B. E. 1996, ApJ, 464, L11

Stern, B. E., \& Svensson, R. 1996, ApJ, 469, L109

Stern, B. E., Poutanen, J., \& Svensson, R. 1997, ApJ, 489, L41

Stern, B. E., Poutanen, J., \& Svensson, R. 1999, ApJ, 510, 312

van der Klis, M. 1989, in NATO ASI C262, Timing Neutron Stars, ed. H. Ögelman \& E. P. J. van den Heuvel (Kluwer), 27 